\title{
Group Structured Reasoning for Coalescing Group Decisions
}

\author{
John Yearwood • Andrew Stranieri
}

Published online: 23 April 2009

(C) Springer Science+Business Media B.V. 2009

\begin{abstract}
In this paper we present the notion of structured reasoning through a model, called the Generic/Actual Argument Model (GAAM). The model which has been used as a computational representation for machine modelling of reasoning and for hybrid combinations of human and machine reasoning can be used as a coalescent framework for decision making. Whilst the notion of structuring reasoning is not new, structured reasoning is advanced as a technique where group consensus on reasoning structures at various levels can be used to facilitate the comprehension of complex reasoning particularly where there are multiple perspectives. For an issue, the approach provides a scaffolding structure for cognitive co-operation and a normative reasoning structure against which group participants can identify points of difference and points in common as well as the nature of the differences and similarities. Intragroup transparency characterized by the ability to recognise points in common and understand the nature of differences is important to the process of coalescing group decisions that carry maximum group support.
\end{abstract}

Keywords Argumentation · Structured reasoning · Practical reasoning · Generic arguments · Decision support

\section{Introduction}

One of the main problems in considering questions of the quality of reasoning (and decision making) is that, for a long time reasoning was associated with 'being logical' and evaluated by the strict criteria of logical formalisms and proof. It was not until

J. Yearwood $(\varangle) \cdot$ A. Stranieri

Centre for Informatics and Applied Optimization, University of Ballarat,

PO Box 663, Victoria, Australia

e-mail: j.yearwood@ballarat.edu.au 
philosophers studied, in depth the character of human reasoning and looked towards alternative representations and logics to capture the nature of informal reasoning that progress has been made in aiding the representation and process of complex reasoning.

The notion of reasoning is closely connected with rationality and the notion of reasons with principles and goals. From the normative logical perspective it is required that reasoning systems be sound and complete but there are other possible views. In many instances, reasoning towards action simply requires 'getting the job done' as distinct from having considered a range of possibilities or all possibilities and selected the best. The logicist approach could be summed up as 'If it's sound, do it' (Doyle 1992). With this approach, issues of the purpose of the reasoning and the value of beliefs and inferences to the reasoner are ignored. The heuristic problem solving approach can also downplay issues of making rational choices unless the heuristics are also used to compare reasoning methods. In his characterization of the knowledge level Newell (1982) formulates his fundamental principle of rationality as "If an agent has knowledge that one of its actions will lead to one of its goals, then the agent will select that action." (Newell 1982, p. 102). Whilst this approach yields solutions it does not compare the different methods for achieving goals.

The notion of reason is fundamental to reasoning and there has been much written on reasons (see for example Raz 1990; Hage 1997). Raz for example, argues that reasons are facts but also allows beliefs to be explanatory reasons in the case of reasons that function as causes and guiding reasons in the case of reasons to act. In our work we use the term reason as in 'reason for relevance' in the sense of a fact or belief that supports the relevance of an item. We also use the term reason more generally in its explanatory sense.

Reasoning occurs in a social context and often, particularly when the reasoning is complex, involves numerous participants with differing views. Normative logical perspectives are less than helpful because the demonstration of soundness and completeness of one participant's view over others rarely occurs. Seldom is evidence unequivocal and open texture, the notion that empirical concepts cannot be defined with completeness prior to their use operates against this approach. Similarly, heuristic problem solving typically describes processes used by a participant to formulate a view and advance assertions that maximize goals but does little to describe or facilitate the reasoning that occurs between participants.

Innovations advanced here that support reasoning between a group of participants include an organisation of reasoning that is sufficiently abstract so as to obtain group consensus on the relevance of all variables without necessitating a commitment by any participant to particular values on any variable. A group of lawyers discussing family law can agree that the length of marriage is a relevant consideration that goes to past contributions for ascertaining the property splits after divorce, without making a commitment to specific assertions about how long the marriage in a case in question was.

In this paper we present the notion of structured reasoning as a normative structure to organise and structure reasoning so that a group of participants can more readily assert their views and compare and contrast them with others. Structured reasoning is presented and exemplified through a model, called the Generic/Actual Argument Model (GAAM) (Yearwood and Stranieri 2006). Structured reasoning is advanced as 
a technique where group consensus on reasoning structures at various levels can be used to facilitate the comprehension of complex reasoning. This paper puts the notion of structured reasoning forward as a useful model in which reasoning may be analyzed but more importantly used to identify points of agreement and points of disagreement among participants of a reasoning community.

The process of thinking, formulating ideas and making decisions is often 'spaghetti-like' or unstructured. Cleaned-up reasoning or explanation might then be what we call well structured reasoning. Techniques for aiding the structuring of reasoning may assist not only its final expression but also the process of reasoning. Structured reasoning as an end product is defined to be a layout of the reasoning possibilities in a tree structure. Each node in the tree admits a defined range of claims, the root of the tree is the top level range of claims which is the goal of the reasoning to resolve. The child nodes of a node are the relevant evidence for reasoning about the parent.

Structured reasoning usually entails: a clearly defined discursive community and purpose; evaluation of the reasoning that is largely based on acceptance of the agreed template (version) for reasoning within the discursive community; agreement within the discursive community on acceptable ways of making inferences within the reasoning structure; a demarcation and explicit identification of the context of the reasoning; the flexibility to combine human with machine inferences; and a shared understanding of the use of concepts in the domain.

Assessing the quality of reasoning in a structured reasoning framework is not based on testing conflicting arguments as in Verheij (1999); Gordon and Karacapilidis (1997); Palmer (1997); Alvarado (1990). Indeed the notion of structured reasoning is to enhance reasoning by establishing a structure that a community accepts. The quality of reasoning is judged against the norm of the structure and the acceptance by the community, not simply on the basis of defeating another argument. Any actual piece of reasoning selects a path through the structure that can be used to identify agreement, similarity, difference and disagreement. It is this facility that can contribute to a coalescing of individual reasoning into group decisions.

The motivation for this paper comes from observations of groups of experts with whom we have undertaken knowledge engineering tasks using the GAAM. It is noticeable and promising that these experts quickly grasp the particular aspect of the GAAM that we call structured reasoning and are able to readily contribute to the development of a structure for reasoning in their domain. For example, although the determination of refugee status according to a United Nations protocol is widely regarded as complex, discretionary and involving few constraints, members of a refugee tribunal were readily able to represent the structure of the reasoning divorced from the assertions in any specific case. Once described, the structure operates as a template to organise claims made by a party and compare them with claims made by applicant's for refugee status, other members and appeal court judges.

The GAAM has been used to model reasoning in copyright law by Stranieri and Zeleznikow (2001a), predict judicial decisions regarding a property split following divorce by Stranieri et al. (1999), support refugee status decision makers by Yearwood and Stranieri (1999), interactive e-commerce by Yearwood et al. (2001), multi-agent negotiation by Avery et al. (2001), determining eligibility for government funded legal aid by Stranieri and Zeleznikow (2001b) and modelling reasoning that critical care 
nurses use Stranieri et al. (2004). The computational model is presented in Yearwood and Stranieri (2006). Two shell programs that implement GAAM ideas are described in Stranieri and Zeleznikow (2001b) and Yearwood and Stranieri (2002a). In this paper we concentrate on capturing the notion and benefits of structured reasoning for group decision making. One of the main concerns of both groups of decision makers and individual decision makers focusing on similar decisions is that of being consistent.

Section 2 looks at some of the aspects of reasoning that pertain to our discussion of structured reasoning later. Section 3 discusses notions of structured reasoning and defines what we mean by structured reasoning. Section 4 provides an outline of argumentation. Section 5 elaborates on the GAAM as a framework for group structured reasoning. Section 6 briefly reviews some technical aspects of the GAAM. Section 7 discusses the advantages of using a structured reasoning approach. Section 8 details reasoning with participants using a generic argument structure and Sect. 9 provides an example of how this contributes to the identification of agreement and difference and how this can assist in coalescing a group decision.

\section{Reasoning}

\subsection{Practical Reasoning}

Practical reasoning is reasoning about what is to be done rather than reasoning about belief which might be called theoretical reasoning. So practical reasoning entails the requirements of doing: actions; series of actions; plans - intentions to initiate a sequence of actions; policies; organizational actions, plans and policies.

Philosophers have been interested in practical reasoning from two points of view: explanation and guidance. Raz (1990), makes the distinction between explanation and guidance but from the point of view of decision support systems to support practical reasoning, we are interested in the general issue of how individuals or groups rationally arrive at and implement decisions about what to do. It is sometimes difficult to tell whether a discourse would be classed as guidance or as explanation and Walton further makes the point that it is difficult to distinguish argument from explanation Walton (1996a).

Practical reasoning is by its nature domain-dependent and only in clearly defined and limited domains of decision making would it make sense to use a single approach to practical reasoning. For example, such a domain is in the medical prescription of drugs. The factors relevant to such a decision are known and few in number. Fox and Das (2000) discuss such a decision making scheme and list the factors: efficacy, side effects, interaction with other drugs being taken, contra indications, patient's past experience with the drug, cost, recommendations by the authorities, local policy and whether a drug is proprietary or generic. This is in contrast to decisions in domains where the number of relevant factors is not known and where the goals of the decision may be multiple or conflicting and where knowledge is incomplete or uncertain.

Practical reasoning is complex because of the possible scope of the decisions that may be addressed but also in the number of types of argumentation schemes that it may involve. Walton (1996b) lists 25 argumentation schemes which may act as the 
vehicle of practical reasoning to a conclusion about what is to be done. His list of schemes includes "argument from...": example, commitment, expert opinion, sign, position to know, verbal classification and evidence to a hypothesis. Other such lists are given in Perelman and Olbrechts-Tyteca (1958) and Grennan (1997). One argumentation scheme involves weighing the points for and the points against a certain action. Indeed the application of these schemes represents a particular form of presumptive reasoning and only gives defeasible support to the courses of action. We say that they are normally only presumptively valid, in the sense that the schemes do not always lead to their conclusion when the premises apply. The presumption can be defeated in the case of there being exceptional circumstances. The 'critical questions' associated with each argumentation scheme serve to direct attention to the conditions that must be satisfied if the presumption is to stand up.

\subsection{Argumentation and Argument Maps}

Verbal reasoning, written reasoning and the formalization of reasoning with logic and even conceptual graph representations have brought us a long way in terms of being able to deal with complex reasoning tasks. However they still require a considerable cognitive load for human understanding of the reasoning or a considerable computing power to carry out the search required to make appropriate inferences. Toulmin's (Toulmin 1958) approach was to look at practical reasoning from the point of view of arguments. He observed that arguments lead not to things that are absolutely true but more to qualified assertions or defeasible conclusions. He relaxed concerns with the formalisms of arguments in terms of formal logic involving premises, connectives, operators and conclusions and focussed on arguments as claims supported probabilistically, by data and warrants. The Toulmin structure of arguments will be discussed in more detail in Sect. 4 but at this stage it is mentioned as the springboard for the work by Robert Horn (1998) on argument maps ${ }^{1}$. Argument maps lay out the structure of arguments in a pictorial or map format. For example Horn has produced a seven chart map of the long running and complex debate on "Can Computers Think?". The maps give a visual representation of the reasoning involved in the debate and allow an overview of the debate, almost at a glance. They provide a representation of the reasoning that is pictorial. It is also claimed that the technique can be used to formulate reasoning and documentation of reasoning within a domain.

Problem Structuring Methods (PSMs) or soft operations research are methods of tackling problematic situations with uncertainty, complexity and conflict faced by a group interested in the problem (Rosenhead 1989). PSMs try to elicit the different interpretations of a problem and reflect them back as a whole to the problem owners so that the richness of the problem may be tackled by all. The richness and complexity of the problem is a function of the number of individuals in the group. They usually involve a facilitator in the process of elicitation and reflection. Some PSMs are: Strategic Options

\footnotetext{
1 Similar work has been done by Yearwood and Stranieri as computational models of reasoning but also as models to assist in the formulation of human decisions (Yearwood and Stranieri 1999; Stranieri et al. 2001).
} 
Development and Analysis (SODA); Cognitive Mapping; Soft Systems Methodology (SSM); Strategic Choice; Robustness Analysis and Dialogue Mapping.

Lin and Shoham (1989) had developed an abstract argumentation system which contained the definition of an argument as a proof tree although Loui (1987) had already defined arguments as graphs. Lin and Shoham introduce the important concept of argument structure and show how to use the resulting framework to capture default reasoning and to formalize inheritance systems. Their aim was the development of formal systems rather than providing maps of complex reasoning.

A different view of argument is in terms of rules and logic. When a rule supporting a conclusion may be defeated by new information it is said that such reasoning is defeasible (Nute 1988). Whenever we chain defeasible reasons to reach a conclusion we have arguments instead of proofs. Chesnevar et al. (2000) have reviewed logical models of argument and provide a comprehensive overview of the area.

Walton's (1996a) book on argument structure presents a new method of argument diagramming. He defines the concept of reasoning structure, generally as $\mathbf{R}=$ $(\mathbf{P}, \mathbf{I}, \mathbf{F})$ where $\mathbf{P}$ is a finite non-empty set of propositions $p_{1}, p_{2}, \ldots, p_{n}, \mathbf{I}$ is a finite set of steps (arcs), $i_{1}, i_{2}, \ldots, i_{m}$ called inference steps and a function $\mathbf{F}: \mathbf{I} \rightarrow \mathbf{P} \times \mathbf{P}$ which maps each step into an ordered pair $\left(p_{i}, p_{j}\right)$ of propositions. An inference step is drawn as a directed line from one proposition to another as shown in Fig. 1 where $\mathrm{X}, \mathrm{Y}, \mathrm{Z}$ and $\mathrm{W}$ would be propositions.

Walton discusses in detail the distinction between linked arguments (where the premises work cooperatively and both are needed, see Fig. 2a) and convergent arguments (where the premises work independently, see Fig. 2b) and strongly puts the case that there is a third option when it is not known whether the argument is linked or convergent. He considers it a serious problem with the conventional method of argument diagramming that the third option cannot be represented. He suggests that the way out of this, in cases where the evidence is incomplete to judge an argument as linked or convergent, is through the use of digraphs and numbering the arrows leading to a common point. In Fig. 1 the two arrows into $\mathrm{Z}$ having the same numbers

Fig. 1 A chain of reasoning

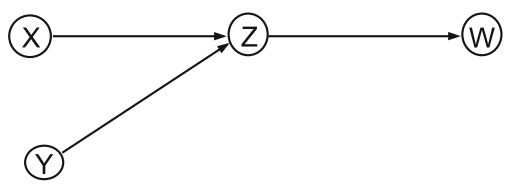

Fig. 2 Argument diagrams

(a)

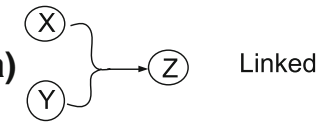

(b)

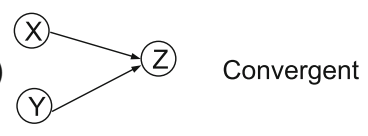

(c)

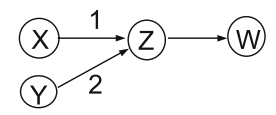


would indicate that both steps are part of the same inference (linked). In contrast, in Fig. 2c the two arrows have different numbers and would indicate two separate steps of inference making the reasoning convergent.

\subsection{Reasoning Structure}

Most approaches to reasoning have been concerned with soundness or with the avoidance of reaching erroneous conclusions, or indeed the strength or force of one line of reasoning (argument) over another. We argue in this paper that it is also constructive to consider the reasoning community and the framework that they establish as the basis for representing reasoning on an issue of concern. Our proposed notion of structured reasoning suggests that reasons are captured as a triple of (factor, position in the structure, context). The factor captures or is a label for the concept or reason, the position captures the nexus to other elements (evidence and conclusions) in the structure and the context captures a whole range of background information. Structured reasoning entails: first an articulation of generalized propositions (or proposition slots) and their relation to each other: secondly, the assignment of values (for each generalized proposition) and agreement on inference procedures that act within this structure and thirdly the construction of individual actual reasoning to a conclusion (value) on the issue. The main contribution of this approach is the understanding of the sources of disagreement or of agreement or similarity that are critical to deciding on whether consensus can be reached and on providing insight into consistency in individual reasoning within the reasoning community.

\section{Structured Reasoning}

We define structured reasoning as an end product, to be a layout or template in a tree structure into which actual pieces of reasoning fit. Each node in the tree holds a position for a range of claims, the root of the tree is the top level set of possible claims which is the goal of the reasoning to resolve. The child nodes of a node in this structure are the slots for the relevant evidence for reasoning about the parent.

As a process, structured reasoning would involve building or using a tree structure for a particular domain. Argument maps and concept maps as described above would provide examples of structured reasoning if the graph is a tree. They do not have the generality to model a range of different reasoning.

Structured reasoning entails:

- a clearly defined discursive community. For example, applicants for refugee status and the decision makers (the case of Reff below);

- a clearly defined discursive community purpose. For example to make transparent decisions on an issue so that differences of opinion between members of the community can readily be identified and communication about the issue is enhanced;

- evaluation of the reasoning is largely based on acceptance of the reasoning within the discursive community; 


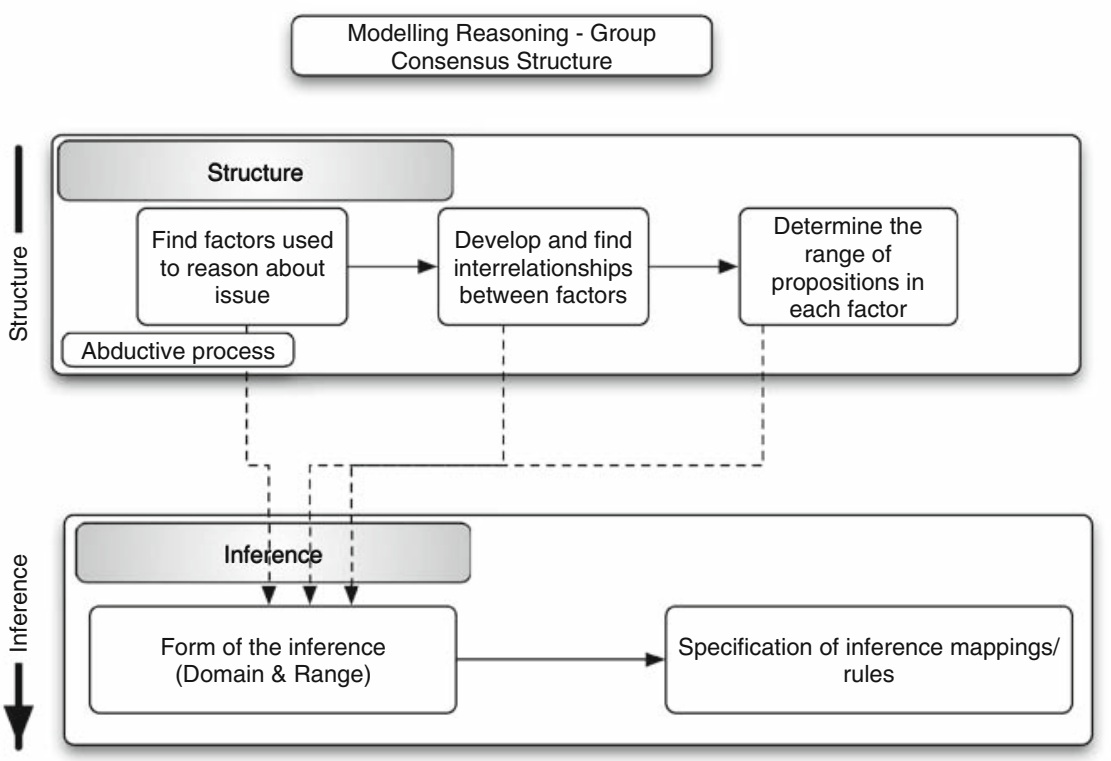

Fig. 3 From structure to inference

- agreement within the discursive community on acceptable ways of making inferences within the reasoning structure;

- a demarcation and explicit identification of the context of the reasoning;

- the flexibility to combine human with machine inferences;

- a shared understanding of the use of concepts in the domain. This is not necessarily at the ontic level usually required by concept sharing in an ontology.

A structured reasoning framework is different from an ontology and from a knowledge representation scheme as neither of these entails a discursive community. The approach facilitates the construction of reasoning that conforms to a normative structure decided by the community. To this extent it assists in making transparent the ways that different reasoning is used. The layout of a piece of reasoning is the first level at which reasoning can be compared and differences between participants' reasoning identified. The next level is the inference level and the focus on inferences is relegated to second position. Figure 3 shows the progression as two major stages, from developing structure to the development of inferences. Structure development entails a form of abduction to find the evidential factors that could be used to reason about the issue, followed by the determination and agreement on how they interrelate and finally the range of plausible propositions in each factor. Development of inference proceeds in two stages, the first being the recognition of the form of each inference procedure from the structuring steps that have preceded and then the acquisition of actual inferences acceptable to the group and reasons (principles or values) that underpin those inferences. 


\section{DATA}

Reff has a real chance of persecution

Relocation within the country of origin is unlikely to lead to protection

\section{BACKING}

High Court of Australia case of Chan Vs Minister of Immigration United Nations refugee Convention Article 9

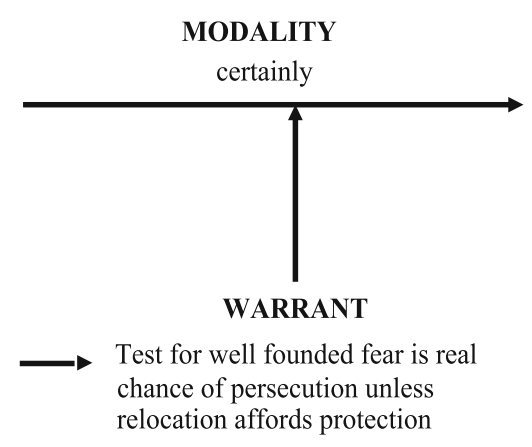

CLAIM

Reff has a well

founded fear of persecution

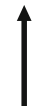

REBUTTAL

Reff is a criminal

Fig. 4 Toulmin argument structure for well founded fear

Whereas ontology is concerned with the articulation of the concepts in a domain and how they relate to each other, it is not concerned necessarily with reasoning. In structured reasoning a concept and its place in the structure together define its reasoning function. Whilst Raz and Hage have defined reasons as facts and beliefs, structured reasoning suggests that reasons are captured possibly as a triple of (factor, position in the structure, context). The factor captures or is a label for the concept or reason, the position captures the nexus to other elements (evidence and conclusions) in the structure and the context captures a whole range of background information. Structured reasoning entails first an articulation of generalized propositions (or proposition slots) and their relation to each other.

A knowledge representation is usually a basis for reasoning that is a formal or semiformal representation. The greater the formality, usually, the greater the separation from a community of users. The success of an underlying knowledge representation is usually evaluated as its ability to adequately capture the knowledge and the ability of inference engines to act on the representation in sound ways. In a structured reasoning framework evaluation of the reasoning is still on the basis of acceptability by the discursive community. At the same time, however the structure readily enables evaluation by making the form explicit.

\section{Argumentation for Structured Reasoning}

Toulmin (1958) concluded that most arguments, regardless of the domain, have a structure that consists of six basic invariants: claim, data, modality, rebuttal, warrant and backing. Every argument makes a claim based on some data. The argument in Fig. 4 is drawn from reasoning regarding refugee status according to the 1951 United Nations Convention relating to the Status of Refugees (as amended by the 1967 United Nations Protocol relating to the Status of Refugees), and relevant High Court of Australia rulings. The claim of the argument in Fig. 4 is the statement that Reff, an applicant to the Refugee Review Tribunal (RRT) ${ }^{2}$ (of Australia) for refugee status, has a well

\footnotetext{
2 The RRT is the body that considers appeals from applicants refused refugee status by the Department of Immigration.
} 
founded fear of persecution. This claim is made on the basis of two data items, that Reff has a real chance of persecution and that relocation within Reff's country of origin is not appropriate. A mechanism is required to act as a justification for why the claim follows from data. This justification is known as the warrant which is, in Fig. 4, the statement that 'The test for well founded fear is real chance of persecution unless relocation affords protection'. The backing provides authority for the warrant and in a legal argument is typically a reference to a statute or a precedent case. The rebuttal component specifies an exception or condition that obviates the claim. Reff may well have a real chance of persecution and relocation is unlikely however the claim that his fear is well founded does not hold as Reff's persecution is due to criminal activities.

Argumentation has been used in knowledge engineering in two distinct ways; with a focus on the use of argumentation to structure reasoning (i.e. non-dialectical emphasis) and with a focus on the use of argumentation to model discourse (i.e. dialectical emphasis). Dialectical approaches typically automate the construction of an argument and counter arguments normally with the use of a non-monotonic logic where operators are defined to implement discursive primitives such as attack, rebut, or accept. Carbogim et al. (2000) present a comprehensive survey of defeasible argumentation.

In general these approaches include a concept of conflict between arguments and the notion that some arguments defeat others. Most applications that follow a dialectical approach represent knowledge as first order predicate clauses, though engage a nonmonotonic logic to allow contradictory clauses. Mechanisms are typically required to identify implausible arguments and to evaluate the better argument of two or more plausible ones.

The preference for one argument over others has been modelled in a variety of ways. Prakken (1993a,b) extends the framework proposed by Poole (1988) by using a concept of specificity. The claim that a penguin flies because it is a bird and all birds fly is less specific than the claim that a penguin does not fly. Preference relations between rules are elicited from experts and explicitly specified in the defeasible reasoning logic described by Antoniou (1997).

A non-dialectical representation facilitated the organisation of complex legal knowledge for information retrieval by Dick $(1987,1991)$. She illustrates how relevant cases for an information retrieval query can be retrieved despite sharing no surface features if the arguments used in case judgements are represented as Toulmin structures. Marshall (1989); Ball (1994) and Loui et al. (1997) have built hypertext based computer implementations that draw on knowledge organised as Toulmin arguments. Hypertext links connect an argument's assertions with the warrants, backing and data of the same argument and also link the data of one argument with the assertion of other arguments. In this way, complex reasoning can be represented succinctly enabling convenient search and retrieval of relevant information.

\section{The Generic/Actual Argument Model for Structured Reasoning}

In trying to acquire and represent knowledge to practically support the tasks of reasoning, justifying and document drafting primarily within legal domains we have modified and generalised the basic Toulmin argument structure. An important aspect 


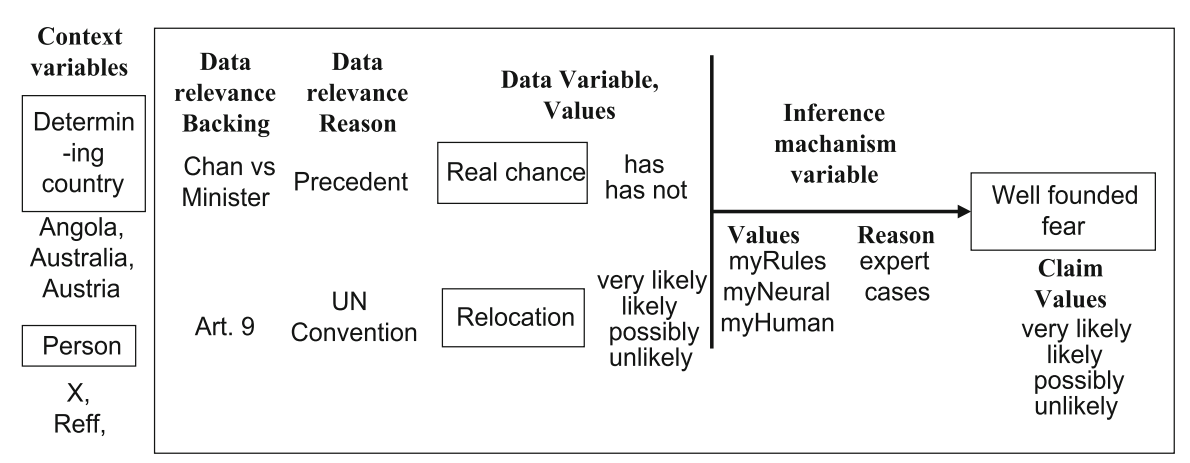

Fig. 5 Generic argument for well founded fear

of our modification is the principle that most arguments, reasoning or justifications within these domains can be represented as a set of generic arguments which link together to form a tree or graph structure. Each generic argument represents a class of actual arguments that may be made and structurally embodies the components that go towards shaping well considered decision making in uncertain domains.

The framework called the Generic Actual Argument Model (GAAM) directly facilitates the structuring of reasoning as arguments are represented at two levels of abstraction; the generic and the actual level. The generic level is sufficiently general so as to represent claims made by all members of a discursive community. All participants use the same generic arguments to construct, by instantiation, their own actual arguments. The generic arguments represent a detailed layout of arguments acceptable to all participants whereas the actual arguments capture a participant's position with respect to each argument. The actual arguments that one participant advances are more easily compared with those advanced by another, in a dialectical exercise because, in both cases the actual arguments have been derived from a generic template that all participants share.

\subsection{Generic Arguments}

Figure 5 illustrates the refugee argument above, as a generic argument. The claim variable has been labelled 'well founded fear' and acceptable values specified. There are three inference procedures known to be appropriate in this example; the first is a rule set that derives from heuristics an immigration expert uses, the second is a neural network trained from past cases and the third is a human inference. This latter inference indicates that a human is empowered with sufficient discretion to infer a claim value from data item values in any way he or she likes.

A generic argument differs from the Toulmin structure in that it is an abstraction that models a group of arguments that make related claims. As a consequence a generic argument includes:

- a variable-value representation for the set of possible claims admitted by the discursive group. For example the variable well founded fear in Fig. 5 is associated with values very likely, likely, possibly and unlikely. A certainty slot not illustrated in the figure is also associated with the claim; 
- a variable-value representation of the data items (with certainty slots) as the grounds on which such claims are made. A data item of one generic argument is the claim of another as the arguments interlink to form a tree structure;

- reasons for relevance of the data items in place of the Toulmin warrant;

- a list of inference procedures that may be used to infer a claim value from data values in place of the warrant;

- reasons for the appropriateness of the inference procedure;

- context variables;

- a claim value reason component.

The data items in each generic argument must be relevant to the claim to the satisfaction of members of the community. A generic argument in a refugee domain is unlikely to include a data item that refers to an applicant's eye colour because a reason for the relevance of eye color to any claim is unlikely to be advanced by any member of the community. The extent to which a community accommodates all possible reasons for relevance is a decision of scope to be made by the community. Some communities will include a data item if any reason for its relevance can be uttered whereas others will impose stricter conditions for relevance. In law, reasons for relevance typically derive from statutes or precedent cases. In scientific arguments factors are deemed relevant by reference to experimental observation or theoretical perspectives.

Figure 5 illustrates two context variables; the Determining country and the Person about which the argument is being made. The respective values are a list of world nations for the Determining Country and the applicant for refugee status, Reff or the more universal $\mathrm{X}$ for the Person. Context variables represent something of the background knowledge that impacts on the generic argument. For example, the context variable Determining country in Fig. 5 represents a scope constraint on the argument. This indicates that an actual argument can be made based on the generic argument however the determining country sets a context for the argument. The context variable is an articulation of the presuppositions that underpin the generic argument.

The context variable can also represent the scope of variables used in the generic argument. For example, the Person context variable will be assigned the value $\mathrm{X}$ for a discourse participant intent on making the more universal argument that relates to well founded fear of anyone. The participant that restricts the argument to Reff does so by setting the context variable to Reff. In general, context is a difficult concept to define. In the framework defined here, context is defined as presupposition and variable scope. However, other definitions can also be accommodated.

The argument template represents knowledge at a very high level of abstraction. There are two levels of instantiation made in applying the template to model arguments within a domain; the generic level and the actual level.

Figure 6 illustrates the Generic Actual Argument Model. Generic arguments exemplified by the well founded fear argument in Fig. 5 are interleaved to form a tree of arguments.

The generic argument is sufficiently general so as to capture the variety of perspectives displayed by members of a discursive community. Actual arguments advanced by members are instantiations of the generic arguments where: 


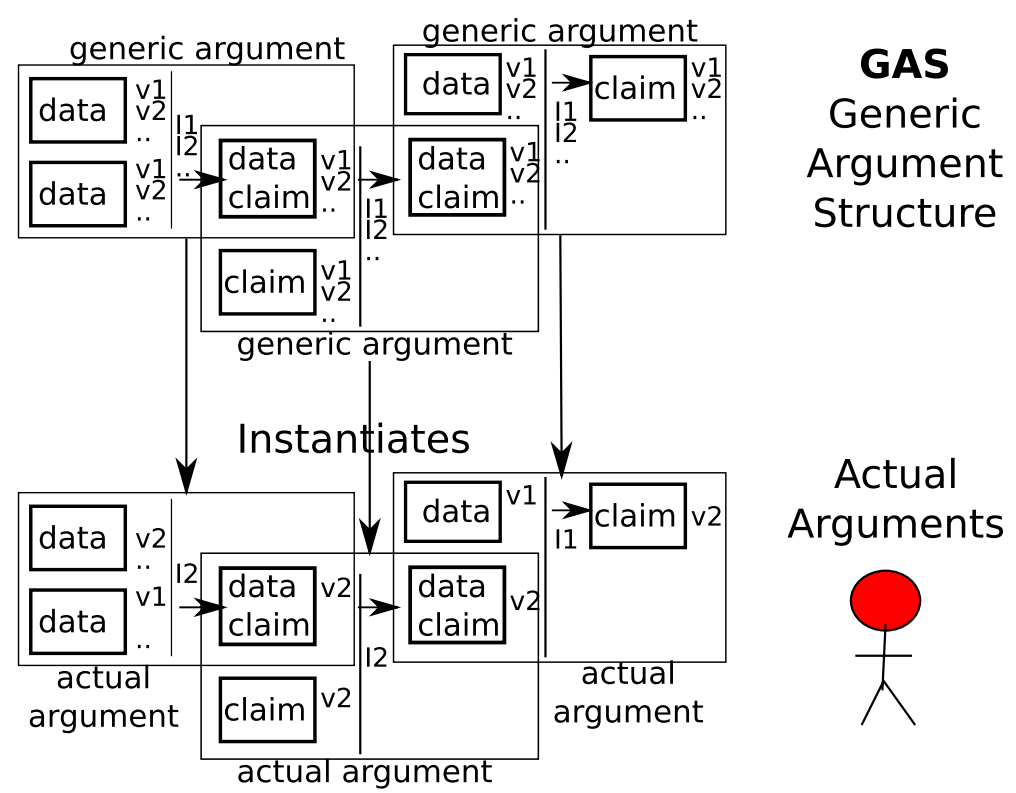

Fig. 6 The generic argument template

- claim, data and context variables are assigned values from those specified in the generic argument;

- a commitment is made to one of the inference procedures listed in the generic structure;

- claim and data variables are assigned certainty values.

\subsubsection{Inference Procedures}

In the GAAM, the Toulmin warrant has been translated to the inference procedure, the reasons for relevance of the data items and the reasons for the inference procedure. This relates to two different roles a warrant can play in an argument. As described above, the warrant indicates a reason for the relevance of a data item and on the other hand the warrant can be interpreted as a rule which, when applied to the data items leads to a claim inference. An inference procedure is an algorithm or method used to infer a claim value from data item values. Under this interpretation, an inference procedure is an operator on data variable values to deliver claim variable values. It is any procedure that will perform a mapping from data items to claim items. A mathematical function, an algorithm, a rule set, a neural network, or procedures yet to be discovered are examples of inference procedures. Many inference procedures can be implemented in software. Thus, they can be automated in computer based systems. However, this need not be necessarily the case for a knowledge engineering framework. Claims can sometimes be inferred from data items by human agents without the explicit specification of an inference procedure. This occurs frequently in discretionary fields of law where, as Christie (1986) notes, decision makers weight and combine relevant 
factors in their own way without articulating precisely how claims were inferred. This situation is accommodated within the Generic Actual Argument framework with the specification of an inference type labelled, simply, human.

Explicitly representing the inference method enables the use of a variety of inference procedures. For example, the method used to infer an assertion in the family law application, Split Up is a rule for some arguments and a neural network for others (Stranieri et al. 1999). Branting (2000) provides a framework that captures legal reasoning using both rules and exemplars. In his framework, rules and exemplars differ primarily in that exemplars are much less abstract than rules and can be used to provide a bridge between the abstract rule descriptions and the specific case descriptions. A knowledge representation framework that separates the inference method from other components is very flexible. For instance, first order predicate logic draws conclusions solely by the operation of inference rules including modus ponens and modus tollens. With non-monotonic logics, the repertoire of inference rules is extended but still tightly embedded into the formalism.

The argumentation framework advanced here not only departs from the Toulmin formulation by distinguishing inference procedure from reasons for relevance but it also represents context explicitly. Further, the generic argument does not represent rebuttal. This is discussed next.

\subsubsection{Rebuttal}

The rebuttal which occurs in the Toulmin structure is not explicitly represented in the GAAM and would be captured within this structure as a different instance argument possibly using a different inference procedure that produces different claim values. The rebuttal is more clearly classified as a dialectical component and it makes sense that it is not explicitly represented in this essentially non-dialectical frame. For instance, discursive participants may create actual arguments as instances of the same generic argument in ways that are quite different from others. Participant A may assert a different claim value than $\mathrm{B}$, yet have perfect agreement on all data item values because a different inference procedure was selected. Any discussion regarding this difference, including exchanges that make the point that the difference constitutes an attack, or exchanges that seek to defend A or B's assertion, or exchanges that seek to identify the stronger argument involve dialectical exchange and are omitted from this nondialectical frame.

It is important to appreciate that the notion of a generic argument can be used to capture a shared understanding about what a core set of factors and arguments in a domain are. In some respects there is some similarity to 'topic theory' Aristotle (1928), (Alexy 1989, p. 21) in that an important aspect in constructing each generic argument is a search for the premises or grounds for that argument. The generic argument represents the results of this search as the data items articulated and their reasons for relevance. These are considered to be 'nearly' complete knowledge about the possible grounds for that argument within the group. As such they would include general exclusionary reasons as described by Raz (1990) which are often the basis for rebuttals. Establishing the generic arguments in a domain provides considerable structure for developing arguments. Engisch (1960) observes that 'reaching a conclusion as 
such gives rise to a minimum of effort; the main difficulty lies in finding premises for it'. We argue that establishing the generic arguments in a domain is an effective part of acquiring, representing, reasoning and providing justification and transparency for decision making.

Figure 5 also includes certainty slots for each data item, claim and inference procedure. These recognize that there is uncertainty in the processes of developing actual arguments. The certainty values are assigned when values are assigned in the process of constructing an actual argument. A generic argument is an agreed approximation to a world but still may only be partial knowledge. We do not explicitly put a certainty or confidence value on a generic argument although we permit generic arguments to change over time.

The structure of generic arguments that describe a domain will not be static. As knowledge within the domain evolves new versions of the generic argument structure will be required. New factors emerge as being relevant to some arguments and new inference procedures may be needed as new legal rules emerge or new cases become precedents. Most actual arguments in a domain are then underpinned by a particular version of the generic argument structure.

The generic arguments within a discursive community or decision making group can be established by engaging participants in a discussion in the development of the generic argument structure (GAS) or through their contributions to a common view of the structure and each participants reasons for relevance (Afshar et al. 2002). The intention is to have participants agree on a structure for reasoning developed from their shared understanding. The open textured nature of many areas of reasoning mitigates against the representation of all arguments in a domain as generic arguments but a large proportion of arguments in many domains can be represented in this way. It is also useful to know when particular actual arguments diverge from instantiations of generic arguments and to detect whether or not they are accepted.

Actual arguments are instances of a generic argument where each data item is assigned a value and an inference procedure is chosen and executed to deliver a value for the claim slot. An actual argument corresponds to a position held by a participant in a discourse. Figure 7 illustrates an actual argument for Reff's well founded fear argument with data values set and the particular inference procedure selected is based on a human myhuman. With human inferencing there is not necessarily a reason for the inference procedure given at the generic level and there is therefore a need to justify the claim value produced. This is represented in the diagram as the claim value reason slot.

The context variable 'person' in the generic argument is instantiated to 'Reff' indicating that the claim only applies to him and not to others. The data item value in Fig. 7 represents the situation that 'Reff is likely to have a well founded fear'.

The claim value reason for this actual argument provides a reason for the specific claim value inferred rather than other claim values. The claim value reason in Fig. 7 expresses a reason for why well founded fear is likely, given the data items and inference procedure selected. If an inference procedure that was a rule set was used, then the rules in this rule set and their appropriateness for reasoning with this data about this claim could possibly be articulated as justification for the claim value. This is what would be called deductive justification by Neil MacCormick (1978). The claim value 


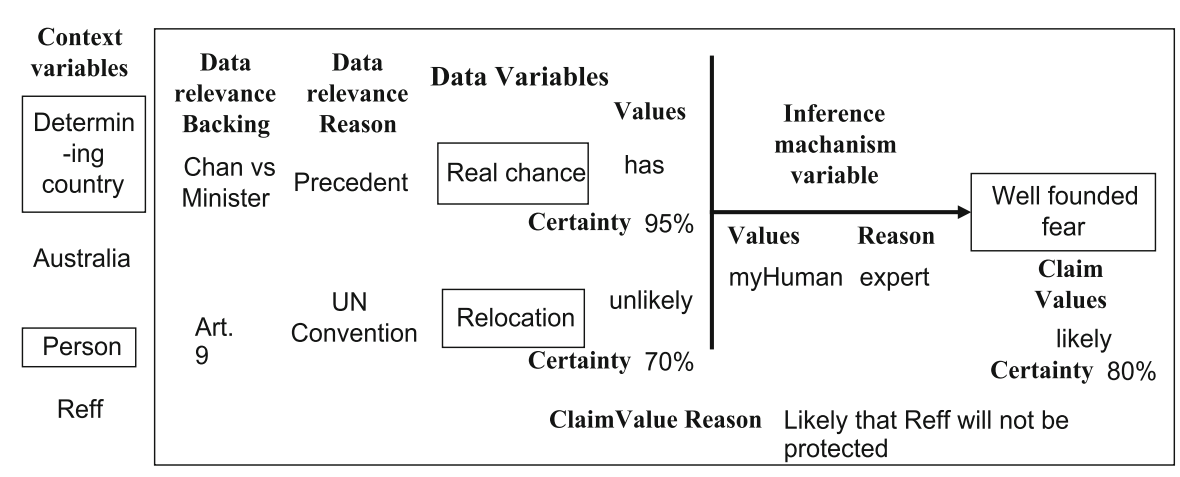

Fig. 7 Actual argument for 'Reff has well founded fear.'

asserted in the figure "likely" needs to be justified and this justification is the claim value reason which is provided at the stage of making an actual argument rather than at the generic argument stage. If the inference procedure is a mathematical function or has mechanisms that are not visible, such as a neural network, then the articulation of a reason for the inference procedure is not an adequate justification of the value. Conceptually, it is more correct to say it is a reason for a particular value that has arisen as a result of the application of an inference procedure.

In the same way that the Generic arguments are chained together to form a Generic Argument Structure (GAS) an actual argument is a chain of propositions that fits within the GAS. Agreements, similarities and differences can be identified between two actual arguments by identifying the position in the structure as well as the inferences chosen. These then provide the objects for negotiation.

Generic and actual argument structures correspond to a non-dialectical perspective. They do not directly model an exchange of views between discursive participants but rather describe assertions made from premises and the way in which multiple claims are organized. Claim variables are inferred using an inference procedure, which may not necessarily be automated, from data item values. The reasoning occurs within a context and the extent to which the data items correspond to true values, according to the proponent of the argument, is captured by certainty values.

The generic argument provides a level of abstraction that accommodates most points of view within a discursive community and anticipates the creation of actual arguments, by participants, as instantiations of a generic argument. However, it is conceivable that, given the open textured nature of reasoning, a participant will seek to advance an actual argument that is a departure from the generic argument. This is a manifestation of discretion and can be realized with the introduction of a new variable (data, claim or context) value, with the use of a new inference procedure or, with a new claim value reason (Stranieri et al. 2000).

This framework including the Generic/Actual distinction, the clear separation of inference procedure from other components and the inclusion of reasons for relevance and context introduces a structure that represents knowledge applicable to a discursive community. 
Situations will arise where an argument needs to be made for which no generic argument exists. In these cases, a new argument specific to that situation is created. Ultimately, the series of actual arguments made in a case is built and represents the full argument in that situation. Some of the arguments are instances of generic arguments, others are newly created.

\section{Defining the GAAM}

The GAAM is a means of specifying generic argument structures to model reasoning within a domain. For a more detailed treatment see Yearwood and Stranieri (2006).

\subsection{A Generic Argument Structure}

A generic argument structure $(G A S)$ is a pair $(\mathcal{C} V, \mathcal{G})$ where $\mathcal{C} V$ is a set of context variables and $\mathcal{G}$ is a connected directed bipartite graph that has two kinds of nodes called, claim slots $\mathcal{C}$ and inference slots $\mathcal{I}$.

Every claim slot $C$ has a prefix $C_{p}$, set of values $C_{v}$ and a suffix $C_{s}$. Each claim slot also has two variables. A variable $r$ which is a place holder for the claim value reason and a variable $c$ which is a place holder for the certainty factor. These are not instantiated at the generic level but at the actual argument level.

Every inference slot $I$ has an arity $(n)$ and a set of pairs of operators and strings $\left(I_{j}, J_{j}\right): j=1, \ldots, k$.

The number of arcs that belong to $I$ is one more than its arity. An inference slot of arity $n$ is represented with $n$ inward arcs and one outward arc. Such an inference slot is called an $n$-ary inference slot. The set of $n+1$ claim slots $<C_{1}, \ldots, C_{n}, C_{n+1}>$ is called the signature of $I$. The set of $n$-ary operators is of the form $I_{j}: C_{1 v} \times \cdots \times$ $C_{n v} \times C V V \rightarrow C_{n+1 v}$ and each operates on the sets of values of the first $n$ claim slots in its signature and the set of context variable values. The strings $J_{j}$ store the justification for the $j^{\text {th }}$ operator.

Every arc from a claim slot to an inference slot is a relevance relation pair $\left(C_{i}, C_{n+1}\right)$ and has two string attributes, $R R$ and $B$ for the reason for relevance and backing.

There is a unique arc from an inference slot to a claim slot.

A generic argument is a GAS that consists of a single inference slot and the claim slots that are attached to its arcs. A full GAS can be formed by connecting individual generic arguments one for each inference slot in $\mathcal{G}$.

An actual argument is an instantiation of a GAS with context variable values, a choice of inference operator (and reason pair) for the inference slot, the assignment of claim values, claim value reasons to the claim value reason variables and the assignment of certainty values to the certainty factor variables.

First note that a claim slot $C_{i}$ defines a set of propositions and the choice of a particular value $C_{v k}$ from the set of values $C_{v}$ defines a proposition (claim): $C_{p} C_{v k} C_{s}$. The actual (atomic) argument for this proposition is then represented as being derived by the application of an inference procedure $I_{h}$ from the inference slot $I$ (leading to the claim slot $\left.C_{i}\right)$ to $n$ values $\left(C_{1 v_{i 1}}, \ldots, C_{n v_{i n}}\right)$ and $C V V$. So, $I_{h}\left(C_{1 v_{i 1}}, \ldots, C_{n v_{i n}}, C V V\right)=$ $C_{p} C_{v k} C_{s}$ where $C_{p}$ is the prefix for $C_{i}$ and $C_{s}$ the suffix. 


\section{Advantages of Structured Reasoning using the GAAM}

The GAAM offers a framework for representing, at two levels, the structure of reasoning within a domain as well as the reasoning itself. The structure manifests as a tree whose root is the top level decision that represents the reasoning goal. This structure has all of the advantages of the Argument maps of Horn (1998) and other versions of TAS used to represent reasoning in a domain. There are, however some important distinguishing features that the GAAM presents that allow it to be advanced as a structured reasoning framework. The first is that it employs the notion of a generic argument to capture a whole class of reasoning rather than reasoning for a particular claim. The second is that it distinguishes between the structure of reasoning and how the reasoning is executed. The third is that the structure of the reasoning is controlled by the notion of relevance. Lastly the separation of the structure from the execution allows the execution to be represented as a range of appropriate inference procedures.

The notion of the generic argument to capture a class of argument using a variable:value representation permits an element of reasoning that parallels the advantage of the use of predicates over single propositions. It entails the coalescing of many specific arguments into a single generic argument with an inference procedure to accommodate the variation in evidence and conclusions.

Having the notion of a generic argument as a fundamental reasoning element ${ }^{3}$ it is then natural to ask how these elements fit together to make up a piece of practical reasoning. One part of the task then is to move from a possibly ill-structured or unstructured task defined as reasoning towards the top level claim. This may be achieved by identifying an on-going knowledge engineering task that involves the noticing and evoking mechanism of Simon (1973) which identifies new considerations, constraints and goals or subgoals. This fitting together of these elements forms the structure that will finally provide a map of the reasoning about a top level claim within a domain. The way in which claims within the structure relate to each other is controlled by the notion of relevance. This is what is commonly referred to as 'adducing the premises' except that it is being done in generality for sets of propositions rather than individual propositions of a dialectical exchange. The effort required by a group to establish such a generic structure proves to be a sound investment in terms of the quality of the final decision as well as the group's satisfaction. This is consistent with findings of Hirokawa and Pace (1983) on group participation and group systematic approach on effective decision-making.

Each data item records its reasons for relevance to the claim with backing given for the reason. As is often the case in employing TAS, the GAAM does not permit internal reasoning about reasons for relevance or backings (see Marshall et al. 1991).

As mentioned in Sect. 2.1 practical reasoning can be complex because of the number of argumentation schemes that it may involve. The GAAM framework supports the representation of multiple individual inference procedures in each generic argument and so can provide the flexibility to capture many different reasoning mechanisms. In

\footnotetext{
3 Birnbaum uses the term argument molecule but this is somewhat different from our notion of generic argument in that it characterizes an argument in terms of a graph of its support and attack relations (Birnbaum 1982).
} 
Fig. 8 Computational diagram of a generic argument

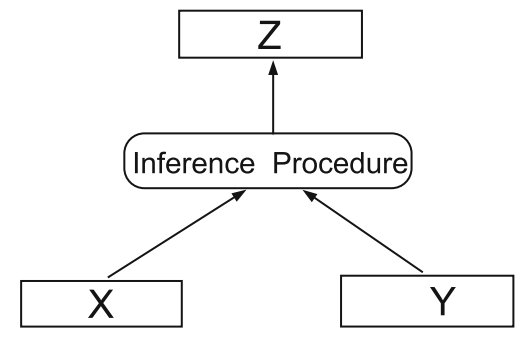

concentrating on first representing the structure and then focussing on the inference from data to claim there is an in-built governance of the notion of mapping from data that are agreed to directly influence the claim in some way.

As well as constraining the generic argument structure by relevance, the domain of each inference procedure (a function) is also appropriately constrained. In contrast to the diagramming technique of Walton, our computational representation of a single generic argument is as shown in Fig. 8 where the conclusion set is linked to the premise sets through an inference slot (a set of inference procedures). This inference procedure representation avoids the technicalities of the linked/convergent dilemma at the generic argument level or at least shifts the problem into the inference procedure slot. The list of inference procedures contained in the inference procedure slot may provide convergent ways of inferring the generic conclusion. Any particular inference procedure may be disjunctive in its use of data items or it may be collective in the use of data items. At the actual argument level the linked/convergent dilemma may also be avoided by not straying from the generic argument structure. In most cases the use of the claim value reason may lead to satisfactory justification given that the structure of the argument has already been captured in a tree structure that will not permit circularity of argument.

The use of the GAAM as a structured reasoning framework generates an actual piece of reasoning with an accepted relevant structure that is explicit. The reasoning process (reasoning step) used at any point can be explained either through the identification of the inference procedure or the claim value reason.

Dialectical exchanges are constrained to the values that claims may take when instantiated from generic arguments. This means that the use of structured reasoning provides a transparent map of the reasoning of each participant or observer of a discourse. The use of structured reasoning can facilitate justice by making explicit not only the agreed reasoning structure that is used within a legal field but also by presenting the actual reasoning within a case in a well structured and explicit form.

The separation of knowledge and inference enables the development of computer systems to flexibly support human reasoning. Within a mapped out reasoning structure particular areas can incorporate automatic inferences based on a variety of inference techniques whilst in other areas inferences can be left to humans.

One of the impediments to the understanding of reasoning as expressed in natural language prose is not only the difficulty in capturing the structure but the need for interpretation of concepts and language that is much less constrained. The GAAM approach overcomes much of this by providing a structure as well as constraining 
the expression to the claims permitted within the GAAM. This creates an arena of discourse that is more transparent to users. The reasoning expressed in this framework is clearly enough defined so that artificial agents can understand each other and a consequence of this is that ambiguity is removed from the reasoning that is represented (see Avery et al. 2001 for details).

To some extent once a generic structure is formulated for a domain the reasoning performed at any step (within a particular argument) amounts to a choice of the claim value based on the accepted data values. The logicist approach would argue that a rational argument would be made on the basis of rules that are consistent and sound. However, economic rationality may guide the choice of claim values. The GAAM supports either of these approaches and it would be permissable for an inference procedure to be specified in terms of sets of preferences of claim values.

\section{Group Reasoning using a Generic Argument Structure}

Within a community or group a GAS provides a constant reminder of the agreed upon, shared understanding and interpretative assumptions. Between communities it provides a public face to the elaboration and explanation as well as the possibility for encouraging participation.

The GAS can be developed by the group in two ways. Participants to a dialogue can collectively deliberate on a structure that will suit as the desired normative structure. Alternatively, a social institution can be charged with the advancement and on-going maintenance of a GAS. Once a GAS is developed for the group then discourse using the structure can proceed.

Discursive communities that have no social institution that can be appropriately charged with the maintenance of a GAS can develop their own GAS. The construction of the generic argument structure can be carried out through structured dialogue between GAAMtalk (a Web version of our argumentation tool) Yearwood and Stranieri (2002b) and participants. The basis of this structured dialogue is the repeated use of a meta generic argument structure. It sets the structure of reasoning and debate for the community on the particular matter for deliberation. As a step toward semi-automating the group construction of a GAS Afshar et al. (2002) describes Consult, a system that enables a community to engage in a Delphi-like communication and a Borda preferendum vote in order to agree on a generic argument structure.

Once the group has developed a GAS, a dialogue between the participants may start anywhere in the tree. This can be viewed as a claim, an inference slot or their actual arguments associated with the claim slot. The object of a dialogue is to identify whether there is agreement on the claim or where in the associated argument, differences may be considered for reconciliation. If there is agreement then the dialogue ends and another dialogue starting at some other point (outside the subtree underneath the agreed claim) in the GAS can occur. If there is no agreement then the participants move to exchanges on each of the claims that are the data for the inference slot. If the claim is a leaf of the GAS then the participants are irreconcilable on data. If they agree on all of the data items then they may differ on the context variable values and in this case they are potentially reconcilable through agreement on context variable 
values otherwise they are potentially reconcilable through agreement on inference. If there are data items that they differ on, then each of these can be used as the starting point for a new dialogue.

Dialogue involving the use of a generic argument structure involves the following steps, formally defined as follows:

Define an actual argument $\mathcal{A}$ associated with an inference slot $I$ in a GAS $\mathcal{G}$ as a tuple $\left(I_{j}, C_{1 i_{1}}, \ldots, C_{n i_{n}}, C_{n+1} v_{i n+1}\right)$.

Within a GAS $\mathcal{G}$ the set of points in common from the arguments of two participants $P_{1}$ and $P_{2}$ is the set $P I C=\left\{C \in \mathcal{C}: C_{v}^{P_{1}}=C_{v}^{P_{2}}\right\}$ and the set of points of difference is the set $P O D=\left\{C \in \mathcal{C}: C_{v}^{P_{1}} \neq C_{v}^{P_{2}}\right\}$.

Definition A dialogue based on a GAS $\mathcal{G}$ is a finite nonempty sequence of moves where move $_{i}=\left(\right.$ Player $\left._{i}, C_{i} \in \mathcal{A}\right)(i>0)$, such that:

(1) $\mathcal{A} \in \mathcal{A}(\mathcal{G})$;

(2) Player $_{i}=P_{1}$ iff $i$ is odd; and Player $_{i}=P_{2}$ iff $i$ is even;

(3) If Player $_{i}=$ Player $_{1}$, then $\mathcal{A}^{1}$;

(4) If Player $i=$ Player $_{2}$, and $C_{n+1 v_{k 2}}^{2}=C_{n+1 v_{k 1}}^{1}$, then $P I C=\left\{C_{n+1}\right\}$ and $P O D=\emptyset$ otherwise $C_{n+1 v_{k 2}}^{2} \neq C_{n+1 v_{k 1}}^{1}$ and $P I C=\emptyset$ and $P O D=\left\{C_{n+1}\right\}$; So, compare data.

(a) If $C_{n+1}^{1}$ is a leaf node of $\mathcal{G}$ then $P_{1}$ and $P_{2}$ are potentially reconcilable on data.

(b) $\quad P I C=\emptyset$ and $P O D=\emptyset$. For each $C_{i} \in \operatorname{signature}(\mathcal{I}) \subset \mathcal{C}, P_{1}$ and $P_{2}$ compare $C_{i v_{k 1}}^{1}$ and $C_{i v_{k 2}}^{2}$. If $C_{i v_{k 1}}^{1}=C_{i v_{k 2}}^{2}$ then $P I C=P I C \cup\left\{C_{i}\right\}$ else $P O D=P O D \cup\left\{C_{i}\right\}$

(c) If $P O D=\emptyset$ then compare context variable value sets, $C V V^{1}$ with $C V V^{2}$. If $C V V^{1} \neq C V V^{2}$ then $P_{1}$ and $P_{2}$ are potentially reconcilable through agreement on context variable values on $C_{n+1}$ of $\mathcal{A}^{i}$ else $P_{1}$ and $P_{2}$ are potentially reconcilable through agreement on inference on $C_{n+1}$ of $\mathcal{A}^{i}$.

(d) If $P O D \neq \emptyset$ then a new dialogue can start for each $C_{i}$ in $P O D$.

The dialogue procedure avoids differences that lie in the sub-tree of an agreed claim. A top level claim is fully discussed when a set of dialogues that covers the GAS has occurred between participants. We will call this a complete dialogue.

This approach to dialogue suggests a particular approach to decision making which allows interaction between participants punctuated with deliberation and the possibility of negotiation and revision. It is also flexible in the selection of arguments that cover the GAS. The procedure can be organised to add claims that participants agree on to their individual commitment stores (Singh 2000). These claims do not need to be revisited. The alternative (monolithic) approach would be to have each participant deliberate and present their complete actual argument that covers the whole GAS. This has the advantage of enforcing consideration of all items in the GAS as well as encouraging complete independence of each participant in the discussion on all arguments in the tree. The former dialogue approach may support less independence and more interaction in the deliberation and group decision process. 


\section{Example}

We consider the case of well founded fear for an applicant, Reff, for Refugee status. The generic argument for well founded fear is shown in Fig. 5. A sample dialogue between participants $\mathrm{X}$ and $\mathrm{Y}$ is as follows:

(1) The discussion commences with $\mathrm{X}$ and $\mathrm{Y}$ accepting to use the generic argument for well founded fear as a template.

(2) X proposes the actual argument claim that Reff very likely has a well founded fear of persecution. Y does not believe this. Y believes that it is unlikely that Reff has a well founded fear of persecution. At this point $\mathrm{X}$ and $\mathrm{Y}$ have, as their point of difference, the claim about Reff's well founded fear.

(3) Y selects a new node to discuss: the node concerning Real Chance of persecution and proposes that Reff has a real chance of persecution. X agrees that Reff has a real chance of persecution. This is a Point in Common. Y selects a new node for discussion.

(4) The other major factor that is used in the determination of well founded fear is whether relocation is possible for Reff. Y proposes that he believes that Reff can be very likely relocated. $\mathrm{X}$ believes that it is unlikely that Reff can be relocated so this is a Point of Difference and they are potentially reconcilable on this data item, Relocation.

(5) $\mathrm{X}$ and $\mathrm{Y}$ have Relocation as a Point of difference and are potentially reconcilable on this point. If either $\mathrm{X}$ or $\mathrm{Y}$ change their claim on relocation this may affect their inference on well founded fear and the dialogue would continue. Further discussion would require agreement on the generic argument with Relocation as its claim node. This generic argument is outside the scope of this paper but would be treated in a similar fashion.

The discussion has identified numerous points of difference and of agreement for both parties to deliberate on. The generic argument structure has provided a template to structure the dialogue without resorting to combative metaphors or unduly constraining the content or flow of the discourse. From the group decision making perspective each participant can focus on points of potential reconciliation and the nature of these can be an aid to achieving a group decision or not.

\subsection{Advantages over other approaches}

The key technical innovation that the structured reasoning approach has is the separation of the structure of the reasoning (defined as the layout of relevant factors) from inference defined as an operation on data values. The case for the value of setting out the map of reasoning has been made but the value of separating out the inferences may not yet be clear. In considering agreement between parties, the separation means that agreement can only occur if the parties agree on the structure and the inferences. This is less likely to occur than on the structure alone. So failing to separate the structure from the inferences means that it is difficult to represent much that a community agrees 
on without recourse to an objective notion of truth (specifying things that every participant must agree on). Separating the knowledge and structure from the inferences also leads to a separation of the decision making task of a group into discussion about the structure/framework and then discussion within the framework which is more closely aligned with inference. From a non-dialectical perspective the way in which claims are laid out and inferred from premises is the object of attention. From the dialectical perspective within a group, the activities of reasoning can be more readily managed if governed by an underlying non-dialectical structure.

Approaches based on logic are concerned with the soundness of the reasoning and this is the norm that is used to evaluate the quality of the reasoning. A structured reasoning approach goes to some trouble to establish an agreed framework for reasoning and permits a range of ways of making inferences. The way in which such a structure is established is described in Yearwood and Stranieri (2002b); Afshar et al. (2002). The quality of the reasoning can be firstly judged on the extent to which it adheres to (or departs from) the framework. If it falls within the norm of the framework then it can be compared with similar or conflicting reasoning from the same evidence. The extent to which difference in reasoning outcomes is acceptable then rests on the acceptance of the inference procedures that have been used. At both levels the divergence can be identified and evaluated against the structure and inference procedures agreed within the framework.

The representation of reasoning in other schemes does not clearly separate the knowledge and the structure of the reasoning from the inference. For example in a classical syllogism both knowledge and inferences are propositions. In FOPL, there are sound inference rules such as modus ponens but there is a mixture of the effect of clauses. For example, the clause man (Socrates) is not distinguished from $\forall(x) \operatorname{man}(x) \rightarrow \operatorname{mortal}(x)$ whereas in the GAAM the latter clause would be distinguished as part of the inference procedure. Semantic networks and conceptual graphs are very similar in their confusion of the two aspects.

Case-based reasoning frequently does not structure the factors in a case and the knowledge base may comprise a set or a structured collection of cases. The inference mechanism based on retrieval of similar cases and their adaptation to the current problem can be viewed as the application of an inference mechanism to the whole set of cases. The clarity of the reasoning to a large extent depends on the case or cases selected as the basis for the new reasoning. The knowledge within a domain is captured as a set of experiences that teach lessons or at least have potential use in reasoning. Although case-bases are often structured and indexed, this structure and indexing is often not explicit. Ashley's (1991) HYPO is an example of a system where a set of partial matching cases is retrieved and organized into a claim lattice. The cases at the top of the lattice share most dimensions with the new case. The cases are then clustered into the most on point cases and potential most on point cases and the case outcomes associated with each cluster examined before a case is selected for use. Other cases are also used in the construction of the 3-ply arguments generated. The experiential knowledge captured by cases can be organized in different ways. It is not clear that an a priori organization can be achieved that maps domain knowledge and signposts the cases to be used for reasoning to particular claims. 
Walton's argument schemes concentrate more on the inference rather than the structure of the reasoning. Each argument scheme acts to classify a way of reasoning and highlight critical questions that must be addressed for the reasoning to be valid. They may act to map critical pathways to reasoning soundly rather than fallaciously but they do not provide assistance with a framework for premises.

Fallacies can be understood as a consequence of failing to make the distinction between the structure and the inferences. For example the argumentum ad populum scheme avoids the consideration of other evidence because the focus is totally on the efficacy of the inference. Furthermore the scheme only leads to fallacious argument if we are looking towards universal truth rather than shared understanding.

The argument diagramming technique of Walton and the diagraph approach of Walton and Batten provide ways of modelling reasoning structure. Walton grapples with the question of linked and convergent arguments and provides the concept of a reasoning structure as a graph. The discussion of a reasoning step in this structure does not go beyond the linked, convergent dilemma. Walton and Batten provide an approach in terms of a diagraph in which the way that rules are applied is made explicit, what demonstrations exist and which premises are not needed in finding a demonstration of an argument. The technique, therefore is able to identify rules, premises and precedence. It could be used to model reasoning in a similar way to the GAAM but uses only rules as inference steps. It is not as prescriptive as the GAAM as cycles are permitted as discussed in Sect. 2. More importantly however, it does not provide a framework that is reusable. The representation, whilst it provides insight, is at the actual argument level. As a framework for structured reasoning it has advantages in that it permits a representation of reasoning patterns that are less directed than the strong notion of structured reasoning that we have proposed. The technique may have application in the untangling stage of developing a reasoning tree structure.

In general, the hypertext approaches (Marshall 1989; Ball 1994; Loui et al. 1997), model the structure of reasoning but do not model the inferences. They permit circularity and their usefulness again is at the level of representing the flow of actual reasoning. Diagrammatic reasoning (Kulpa 1994) can encapsulate knowledge and reasoning in such a tightly coupled way that it provides very efficient and convincing reasoning. The difficulty is that the production of diagrams of this nature requires very careful insight.

Bart Verheij's (1999) ArguMed is an argument assistance system based on the defeasible logic DEFLOG. The ArguMed system uses the notion of a warranted dialectical argument. A dialectical argument is one in which counterarguments (based on undercutting exceptions) are incorporated. The system allows warrants, both for argument steps (the reasons that support conclusions) and for undercutters (for the exceptions that block the connections between a reason and the conclusion). Step warrants express that a particular statement can be adduced as a reason for another statement. Undercutter warrants express that a particular statement provides an exception that breaks the connection between a reason and conclusion. Whether a dialectical argument justifies its conclusion depends on the structure of the argument that is on the reasons, conclusions, exceptions and warrants that occur in it, and on the way they are related. The system automatically determines the justification status of an argument. The notions of reason, conclusion, step warrant and reason for a step warrant are 
respectively similar to the GAAM's notions of data item, claim, inference and reason for inference procedure. The exceptions, like the Toulmin rebuttal make the argumentation defeasible. The undercutter warrants have no counterpart in Toulmin's scheme but would correspond to an inference to a contrary claim at the actual argument level in the GAAM. Toulmin does not give an explicit characterisation of the justification status of statements and the GAAM does not necessarily attempt to do this in an absolute sense but relative to the norm of the group's GAS. This approach is much more explicit for dialectic exchanges and is free in terms of adducing reasons (backwards) or inferring conclusions (forwards) but is not as explicit in structuring reasoning. In the GAAM, adduction takes place at the first stage when the group establishes the structure then individual inference to conclusions can proceed subsequently within the structure.

Gordons's Zeno (Gordon and Karacapilidis 1997) and Loui's Room 5 (Loui et al. 1997) are issue based argumentation systems. Like the GAAM these systems focus on the justification of an initial central issue. Both of these systems allow reasons to be chained and a distinction is made between reasons for and against a conclusion. In Zeno, weighing the conflicting reasons determines which conclusion is justified. Zeno, like ArguMed allows the determination of the status of statements and arguments by the system. Neither of these systems assist participants in structuring the reasoning to the extent that a GAAM based model would.

James Palmer (1997) gives a structural analysis of legal merit arguments. He demonstrates that behind their apparent diversity lies a remarkable uniformity in terms of: the small number of argument-bites or stock narratives upon which these arguments are based; and the recurring interrelationships of support and opposition that exists between them. This structural analysis is useful in its own right and supports the incorporation of reasons for relevance in the GAAM. The interrelationships of support and opposition are not captured within the GAAM but would be captured by a dialectical model based on the GAAM.

Sergio Alvaradi in developing the system OpEd uses a classification of arguments into argument units based on relations of support and attack (Alvarado 1990). This argument unit structure plays a vital part in the understanding of editorial text. He argues that this structure is domain independent and provides both a computational and arguably a useful general approach to considering and structuring arguments. The structuring used in this approach is focused on success or failure in terms of goals and the possible reasoning of opponents. This is in contrast to our structured reasoning approach which can be seen as providing a template for domain specific reasoning structure to be developed collaboratively and in a coalescent manner.

Within the GAAM a generic argument structure is similar to an ontology in that it represents a shared understanding of the knowledge in that domain. The ontology/inference engine distinction is becoming increasingly accepted as seen with the development of the Ontology Inference Layer (OIL) (Fensel et al. 2000). OIL permits reasoning using description logic within the ontological specification but is a layer for inference engines to act on. The separation of the knowledge and the inference within the GAAM can be viewed similarly but there is not a single inference engine envisaged. The GAAM permits the use of appropriate inference procedures in each generic argument. 


\subsection{Advantages of the generic structure}

The process of developing the generic structure of reasoning in a domain involves arriving at a tree that experts within the domain agree on. Currently, methods of acquiring the structure efficiently are being developed (Afshar et al. 2002). Once the structure for a domain is developed it can change over time. The usual way in which this happens is through an assimilation of deviant cases. That is through human recognition of a general need to amend the current structure because of particular new ways in which cases have been handled. Use of the GAAM framework allows not only the collection of reasoning instances that build up a large repository of community knowledge but the possibility of automatically discovering shifts in usage or new patterns of usage. The data can also be used to update and offer alternative inference procedures or support mechanisms for human inferences.

The generic structure derives its power from two important elements of abstraction: the separation of the layout of factors from the inference and the formulation of the structure in abstract terms that can be instantiated to represent a multitude of actual arguments. It is this generality that permits the reuse of the structure.

\section{Conclusion}

The notion of structured reasoning described is based on: the generalised reasoning slots described; selecting these based on group consensus of the relevant factors involved; their inferential relationship to each other; and the possible ways of inferring particular propositions within these slots.

The GAAM acts as a structured reasoning framework for group decision making by providing the following features:

- Abstract arguments - providing an effective notion of generic argument that acts as a fundamental reusable element of reasoning structure.

- Structure based on relevance - providing a framework that represents and maps the structure of reasoning as a tree which is controlled by the notion of relevance as determined by the group

- Separation of structure and inference - in the bipartite graph the methods of inference within a piece of reasoning are separate from the data/claims and together comprise the structural layout of the reasoning.

- Flexible dialectical support - supports multiple actual arguments that exist within a group. These can be developed as purely computational reasoning elements, as computational elements which can be dialectically elaborated or as pure dialectical elements

The structured reasoning approach develops the task of generating explicit, justificatory reasoning within a domain in four stages:

- Structure development - The mapping out of the generic argument tree as a layout of the shared understanding by the discursive community of the way in which generic arguments in the domain fit together based on relevance. This moves the problem that might be classed as ill-structured to one that is well structured. The generality of the structure permits reuse. 
- Detailing the claim slot nodes - Capturing the full detail of the claims, values, reasons for relevance and backings for each generic argument.

- Detailing the inference slots - Determining the inference procedures for each generic argument and constructing them.

- Actually reasoning - The instantiation of generic arguments with values and possibly claim value reasons to present an actual piece of reasoning. This reasoning is then well structured as it fits into the agreed well structured template or map.

Overall, the representation of reasoning and its context can be fully explicit and transparent in the group/community developed GAS. The GAS then permits a range of dialectical arguments from group participants and can be used to support group decision making by allowing participants to identify the basis of their differences and similarities. The main contribution then is the identification, for participants, of whether they differ on claims, inferences or contextual information and the exact nature of their difference. From the computational point of view the structure is like a heuristic that defines the search space very efficiently. So for groups of software agents trying to reach a group decision (which may be defined as some form of consensus) the points to be negotiated become very clear. From the human reasoning view point it acts in at least two ways: as an explicit structure that scaffolds cognitive co-operation between group members and as a reference or norm in the same way that a map does. The focus is not on reasoning as an adversarial exercise but as a cooperative enterprise where agreements as well as disagreements in complex decisions can be identified understood and negotiated.

Acknowledgements This research was supported by the Australian Research Council

\section{References}

Afshar F, Yearwood J, Stranieri A (2002) Capturing consensus knowledge from multiple experts. In: Bramer M (ed) Research and development in intelligent systems XIX. Springer-Verlag, London

Alexy R (1989) A theory of legal argumentation: the theory of rational discourse as theory of legal justification. Clarendon Press, Oxford

Alvarado SJ (1990) Understanding editorial text: a computer model of argument comprehension. Kluwer Academic Publishers, Norwell

Antoniou G (1997) Nonmonotonic reasoning with incomplete and changing information. MIT Press, Cambridge, Mass

Aristotle (1928) The works of Aristotle, i. Logic (trans: Pickard-Cambridge WA). Chap. Topica, Clarendon Press, Oxford, p 100

Ashley KD (1991) Reasoning with cases and hypotheticals in HYPO. Int J Man-Machine Stud 34(6):753796

Avery J, Yearwood J, Stranieri A (2001) An argumentation based multi-agent system for eTourism dialogue. In: Proceedings first international workshop on hybrid intelligent systems HIS 2001. Adelaide, Australia, pp 497-512

Ball WJ (1994) Using Virgil to analyse public policy arguments: a system based on Toulmin's informal logic. Social Sci Comput Rev 12(1):26-37

Birnbaum L (1982) Argument molecules: a functional representation of argument structure. In: Proceedings of the national conference on artificial intelligence, American Association for Artificial Intelligence. AAAI, Pittsburg, PA, pp 63-65

Branting LK (2000) Reasoning with rules and precedents-a computational model of legal analysis. Kluwer Academic Publishers, Dordrecht 
Carbogim D, Robertson D, Lee J (2000) Argument-based applications to knowledge engineering. Knowl Eng Rev 15(2):119-149

Chesnevar CI, Maguitman AG, Loui RP (2000) Logical models of argument. ACM Comput Surv 32(4):337-383

Christie GC (1986) An essay on discretion. Duke Law J 35(5):747-778

Dick JP (1987) Conceptual retrieval and case law. In: Proceedings of the first international conference on artificial intelligence and law. ACM Press, pp 106-115

Dick JP (1991) A conceptual, case-relation representation of text for intelligent retrieval. Ph.D. thesis, Department of Computer Science, University of Toronto

Doyle J (1992) Rationality and its roles in reasoning. Comput Intell 8(2):376-409

Engisch K (1960) Logische Studien zur Gesetzesanwendung, 2nd edn. Heidelberg Press, Heidelberg

Fensel D, Horrocks I, Harmelen FV, Decker S, Erdmann M, Klein M (2000) OIL in a nutshell. In: Dieng R (ed) Proceedings of the 12th European workshop on knowledge acquisition, modeling, and management (EKAW'00). Springer-Verlag, pp 1-16

Fox J, Das S (2000) Safe and sound: artificial intelligence in hazardous applications. AAAI Press/MIT Press, Menlo Park, CA/Cambridge

Gordon TF, Karacapilidis NI (1997) The zeno argumentation framework. In: Proceedings of the sixth international conference on artificial intelligence and law. ACM Press, pp 10-18

Grennan W (1997) Informal logic: issues and techniques. McGill-Queen's University Press, Montreal

Hage JC (1997) Reasoning with rules: an essay on legal reasoning and its underlying logic, vol 27 of law and philosophy library. Kluwer Academic Publishers, Dordrecht, The Netherlands

Hirokawa R, Pace R (1983) A descriptive investigation of the possible communication-based reasons for effective and ineffective group decision-making. Commun Monogr 50:363-379

Horn RE (1998) Using argumentation analysis to examine history and status of a major debate in artificial intelligence and philosophy. In: Proceedings of the fourth international conference of the international society for the study of argumentation, SIC SAT

Kulpa Z (1994) Diagrammatic representation and reasoning. Mach Graph Vis 3(1/2):77-103

Lin F, Shoham Y (1989) Argument systems: a uniform basis for nonmonotonic reasoning. In: Proceedings of the first international conference on knowledge representation and reasoning. Morgan Kaufmann Publishers, pp 245-255

Loui RP (1987) Defeat among arguments: a system of defensible inference. Comput Intell 3(3):100-106

Loui R, Norman J, Altepeter J, Pinkard D, Craven D, Lindsay J, Foltz M (1997) Progress in room 5: a testbed for public interactive semi-formal legal argumentation. In: Proceedings of the sixth international conference on artificial intelligence and law. ACM Press, New York, pp 207-214

MacCormick N (1978) Legal reasoning and legal theory. Oxford University Press, Oxford

Marshall CC (1989) Representing the structure of legal argument. In: Proceedings of the second international conference on artificial intelligence and law. ACM Press, USA, pp 121-127

Marshall CC, Halasz FG, Rogers RA, Janssen WC Jr. (1991) Aquanet: a hypertext tool to hold your knowledge in place. In: Proceedings of the third annual acm conference on hypertext. ACM Press, pp 261-275

Newell A (1982) The knowledge level. Artif Intell 18(1):87-127

Nute D (1988) Defeasible reasoning. In: Fetzer JH (ed) Aspects of artificial intelligence. Kluwer Academic Publishers, Norwell, MA, pp 251-288

Palmer J (1997) Legal merit arguments, legal semiotics and the design of legal knowledge-based systems. In: Proceedings of the sixth international conference on artificial intelligence and law, ICAIL'97, pp 198-205

Perelman C, Olbrechts-Tyteca L (1971/1958) The new rhetoric: a treatise on argumentation (trans: Wilkinson J, Weaver P). University of Notre Dame Press, Notre Dame/London. (French language original first published in 1958)

Poole DL (1988) A logical framework for default reasoning. Artif Intell 36:27-47

Prakken H (1993a) A logical framework for modelling legal argument. In: Proceedings of the fourth international conference on artificial intelligence and law. ACM Press, New York, pp 1-9

Prakken H (1993b) Logical tools for modelling legal argument. Ph.D. thesis, Vrije University, Amsterdam, The Netherlands

Raz J (1990) Practical reason and norms, 2nd edn. Oxford University Press, Oxford

Rosenhead J (1989) Rational analysis for a problematic world: problem structuring methods for complexity, uncertainty and conflict. Wiley, Chichester 
Simon HA (1973) The structure of ill structured problems. Artif Intell 4:181-201

Singh MP (2000) A social semantics for agent communications languages. In: Dignum F, Chaib-draa B, Weigand $\mathrm{H}$ (eds) Proceedings of the international joint conference on artificial intelligence (IJCAI-99), Workshop on agent communication languages. Springer

Stranieri A, Zeleznikow J (2001a) Copyright regulation with argumentation agents. Inf Commun Technol Law 10(1):123-137

Stranieri A, Zeleznikow J (2001b) WebShell: the development of web based expert system shells. In: Research and development in expert systems XVIII. Proceedings of ES2001- The twenty-first SGES international conference on knowledge based systems and applied artificial intelligence. Springer Verlag, London, pp 245-258

Stranieri A, Zeleznikow J, Gawler M, Lewis B (1999) A hybrid rule-neural approach for the automation of legal reasoning in the discretionary domain of family law in Australia. Artif Intell Law 7(2-3):153-183

Stranieri A, Yearwood J, Meikle T (2000) The dependency of discretion and consistency on knowledge representation. Int Rev Comput Law Technol 14(3):325-340

Stranieri A, Zeleznikow J, Yearwood J (2001) Argumentation structures that integrate dialectical and nondialectical reasoning. Knowl Eng Rev 16(4):331-358

Stranieri A, Yearwood J, Gervasoni S, Garner S, Deans C, Johnstone A (2004) Web-based decision support for structured reasoning in health. In: Karin W, Cesnik B (eds) Proceedings of the combined conferences of the twelfth national health informatics conference, pp 180-185

Toulmin S (1958) The uses of argument. Cambridge University Press, Cambridge

Verheij B (1999) Automated argument assistance for lawyers. In: Proceedings of the seventh international conference on artificial intelligence and law, ICAIL'99, pp 43-52

Walton DN (1996a) Argument structure: a pragmatic theory. Toronto studies in philosophy. University of Toronto Press, Toronto

Walton DN (1996b) Argumentation schemes for presumptive reasoning. Lawrence Erlbaum Associates, Mahwah, NJ

Yearwood J, Stranieri A (1999) Integration of retrieval, reasoning and drafting for refugee law: A third generation legal knowledge based system. In: Proceedings of seventh international conference on artificial intelligence and law, ICAIL'99, pp 117-125

Yearwood J, Stranieri A (2002a) An argumentation shell for supporting the development and drafting of legal documents. Inf Commun Technol Law 11(1)

Yearwood J, Stranieri A (2002b) Generic Arguments: a framework for supporting online deliberative discourse. In: Enabling organisations and society through information systems, Proceedings of the thirteenth Australasian conference on information systems (ACIS'2002), pp 337-346

Yearwood J, Stranieri A (2006) The generic/actual argument model for practical reasoning. Decis Support Syst 41(2):358-379

Yearwood J, Stranieri A, Avery J (2001) Negotiation and argumentation based agents to facilitate eCommerce. In: Proceedings of the International conference on advances in infrastructure for electronic business, Science and education on the internet, SSGRR2001, pp 100-109 